\title{
Synthesis and crystal growth of Rh(IV) oxides with double perovskite structure
}

\author{
Tamara Holub ${ }^{1}$, Ryan Morrow ${ }^{1}$, Anja Wolter-Giraud ${ }^{1}$, Bastian Rubrecht ${ }^{1}$, Bernd Büchner ${ }^{1,2}$ \\ ${ }^{1}$ Leibniz Institute for Solid State and Materials Research Dresden IFW, Dresden D-01069, Germany \\ ${ }^{2}$ Institute for Solid State Physics, Technische Universität Dresden, Dresden D-01069, Germany
}

t.holub@ifw-dresden.de

Transition metal oxides with the perovskite crystal structure constitute a class of materials long considered highly interesting for research in structure-property relationships and materials design. In this work we consider the $B$ cation rock salt ordered double perovskite structure, with general formula $A_{2} B B^{\prime} \mathrm{O}_{6}$. We are interested in the magnetically frustrated fcc $B^{\prime}$ cation sublattice, where on the $B^{\prime}$ position we have placed $\mathrm{Rh} 4 \mathrm{~d}^{5}$ as a reduced SOC analogue in the context of our previous iridate double perovskite work.

We have synthesized $\mathrm{La}_{2} B \mathrm{RhO}_{6}(B=\mathrm{Zn}, \mathrm{Mg})$ compounds as powders and single crystals that form in the $P 2_{1} / n$ space group. Their structure and properties have been characterized with a combination of XRD, SQUID magnetometry, and heat capacity. Interestingly, the materials exhibit no long range order to very low temperatures, and may host an exotic magnetic ground state.

Keywords: Crystal growth, double perovkite, magnetic flustration 